\title{
Influence of metronidazole resistance on efficacy of quadruple therapy for Helicobacter pylori eradication
}

\author{
R W M van der Hulst, A van der Ende, A Homan, P Roorda, J Dankert, G N J Tytgat
}

\begin{abstract}
Background-Metronidazole-containing eradication therapies are less effective for metronidazole resistant Helicobacter pylori. Although early data suggested improvement of the efficacy of bismuth triple therapy after the addition of acid suppressives, these findings were based on studies with small numbers of patients, incomplete post-eradication follow up, or omission of pretreatment susceptibility testing.

Aims-To study the efficacy of quadruple therapy in the Amsterdam area, where the efficacy of bismuth triple therapy has been proved to be affected by metronidazole resistance.

Patients and methods-Eighty two consecutive dyspeptic $H$ pylori positive patients with either metronidazole susceptible (group I) or metronidazole resistant $H$ pylori strains (group II) received quadruple therapy for one week: omeprazole $20 \mathrm{mg}$ twice daily; colloidal bismuth subcitrate $120 \mathrm{mg}$ four times a day; tetracycline $500 \mathrm{mg}$ four times a day; metronidazole $500 \mathrm{mg}$ three times a day. Susceptibility to metronidazole was determined by the E-test.
\end{abstract}

Results-Intention to treat analysis showed that $H$ pylori infection had been cured in $42 / 43$ patients $(98 \%)$ in group I and $32 / 39$ patients $(82 \%)$ in group II $(p=$ 0.02).

Conclusion-The efficacy of quadruple therapy is significantly impaired in patients infected with metronidazole resistant $H$ pylori. Therefore a nonmetronidazole-containing regimen should preferably be used in areas known to have a high prevalence of pretreatment metronidazole resistance.

(Gut 1998;42:166-169)

Keywords: quadruple therapy; metronidazole resistance; Helicobacter pylori; gastritis; duodenal ulcer disease

The pathogenic role of Helicobacter pylori in chronic active gastritis and the association with duodenal ulcer disease in $95-99 \%$ of patients and most gastric ulcer patients are well established. ${ }^{1-3}$ Therefore the 1994 NIH consensus development conference recommended attempted eradication of $H$ pylori in all patients with documented peptic ulcer disease. ${ }^{4}$ Currently used eradication regimens show efficacy ranging from 80 to $95 \% .^{5}$ Quadruple therapy comprising a proton pump inhibitor (PPI) in combination with bismuth triple therapy appears to produce the highest eradication rates (96\%; range $92-97 \%) .^{5}$

Since resistance to metronidazole considerably affects the efficacy of bismuth triple therapy (which contains metronidazole), ${ }^{5-7}$ and the latter is the basis for the quadruple regimen, it is questioned whether the effectiveness of quadruple therapy (bismuth triple + PPI) will also be impaired in cases of resistance to metronidazole.

Limited date are available suggesting that the addition of acid suppressives to the bismuth triple therapy regimen (quadruple) overcomes the deleterious effect of metronidazole resistance to its efficacy. ${ }^{89}$

To explore this problem further in a large consecutive cohort of dyspeptic patients, we studied the efficacy of quadruple therapy in the Amsterdam area, in which the prevalence of metronidazole resistance is high and the efficacy of bismuth triple therapy has been proved to be impaired in cases of metronidazole resistance. ${ }^{6} 10$

\section{Patients and methods}

PATIENT SELECTION

Consecutive dyspeptic $H$ pylori positive patients referred to our centre for diagnostic upper gastrointestinal tract endoscopy were enrolled in the study after verbal informed consent had been obtained. Patients were excluded if they were younger than 18 or older than 75 , if they had an allergy to one of the drugs, if they had current complications of peptic ulcer disease-for instance, active upper gastrointestinal tract bleeding or perforation, or if they needed maintenance treatment with omeprazole. Additional exclusion criteria were liver or kidney disease, severe cardiac or pulmonary disease, alcoholism, drug abuse, or any other condition associated with poor patient compliance, suspected or confirmed malignancy, pregnancy, and breast feeding. In addition, patients who were already being treated with omeprazole, bismuth compounds, antibiotics, or investigational drugs during the 30 days before the pre-entry endoscopy were excluded.

The medical ethics committee of the institution approved the study design.

STUDY DESIGN

Patients harbouring either metronidazole susceptible (met-S) or metronidazole resistant (met-R) $H$ pylori received oral omeprazole 20 
Table 1 Patient characteristics and eradication results after quadruple therapy in patients infected with either metronidazole resistant or metronidazole susceptible Helicobacter pylori

\begin{tabular}{lll}
\hline & Met-S $(n=43)$ & Met $-R(n=39)$ \\
\hline Median (range) age (y) & $55(29-52)$ & $46(25-48)$ \\
Sex (M/F) & $28 / 15$ & $19 / 20$ \\
Race (white/non-white) & $31 / 12$ & $23 / 16$ \\
Peptic ulcer disease/functional dyspepsia & $19 / 24$ & $13 / 26$ \\
Compliance < 75\% & 0 & 0 \\
Treatment withdrawn because of side effects & 0 & 0 \\
Eradication rate & $42 / 43(98 \%)$ & $32 / 39(82 \%)^{\star}$ \\
$95 \%$ Confidence interval & 0.88 to 1.0 & 0.67 to 0.97 \\
\hline
\end{tabular}

${ }^{\star} \mathrm{p}=0.02$ compared with met-S

mg twice a day, colloidal bismuth subcitrate $120 \mathrm{mg}$ four times a day, tetracycline $500 \mathrm{mg}$ four times a day, metronidazole $500 \mathrm{mg}$ three times a day for one week. The study drugs were taken during meals and late in the evening.

After the end of the study period, patients with persisting dyspeptic symptoms received $\mathrm{H}_{2}$ receptor antagonists, since these drugs do not have any suppressive activity against $H$ pylori.

Patient compliance was assessed by counting the returned study drugs eight days after the start of the therapy. Intake of less than $75 \%$ of the prescribed number of capsules was considered an inadequate compliance, but was not an exclusion criterion for the intention to treat analysis. During the treatment period, patients were asked to refer to their study doctor if they had side effects.

\section{H PYLORI ASSESSMENT}

$H$ pylori assessment was performed before and 4-6 weeks after the end of the treatment by histopathological examination and culture of gastric biopsy specimens.

For histopathological examination, haematoxylin/eosin stained antrum $(\mathrm{n}=2)$ and corpus $(n=2)$ biopsy samples were examined. No additional special staining was performed. Gastric biopsy samples from antrum $(n=1)$ and corpus $(n=1)$ were cultured on selective and non-selective blood agar plates under microaerophilic conditions. Gram negative and oxidase, catalase, and urease positive spiral or curved rods were identified as $H$ pylori. The histopathologist, microbiologist, and gastroenterologist were blinded to each others results.

$H$ pylori infection was considered to be present if culture and/or histopathological results were positive, and was defined as being cured if $H$ pylori were absent on both culture and histopathological examination.

\section{ANALYSIS OF H PYLORI SUSCEPTIBILITY TO}

TETRACYCLINE AND METRONIDAZOLE

Minimum inhibitory concentrations (MICs) were determined by the E-test (AB Biodisk, Sweden). Frozen primary cultures from both antrum and corpus were inoculated on fresh Columbia agar plates containing $7 \%$ horse blood and cultured under microaerophilic conditions at $37^{\circ} \mathrm{C}$. After three days the grown $H$ pylori were collected using a cotton swab and resuspended in $2 \mathrm{ml}$ Dulbecco's modified Eagle's cell culture medium. From this suspension $100 \mu \mathrm{l}$, containing $10^{7}-10^{8}$ colony forming units/ml, was flooded on Colombia agar plates containing $7 \%$ horse blood. According to the instructions of the manufacturer, E-test strip was placed on the agar when the surface of the plate was dry. All plates were incubated at $37^{\circ} \mathrm{C}$ under microaerophilic conditions for three and five days. In the E-test the MIC was defined as the concentration on the E-test strip closest to the point of intersection with growth on the plate. Colonies growing within the zone of growth inhibition of the bacterial lawn were either resuspended in $100 \mu \mathrm{l}$ Dulbecco's modified Eagle's medium and directly reassessed, or subcultured for another three days on blood agar plates before reassessment as described above. ${ }^{11} H$ pylori strains with an MIC value $>8 \mu \mathrm{g} / \mathrm{ml}$ were considered to be resistant, whereas those with an MIC value < $4 \mu \mathrm{g} / \mathrm{ml}$ were considered to be susceptible to metronidazole. ${ }^{611}$

\section{STATISTICAL ANALYSIS}

Treatment outcome was assessed by intention to treat analysis. From previous studies, eradication efficacy is estimated to be $95 \%$ for both met-R and met-S treatment groups. Assuming a difference of $20 \%$ in eradication efficacy between the two treatment groups to be clinically relevant, 39 patients should be included per treatment group, based on a two sided test with a power of $80 \%$ and an alpha error of $5 \% .^{12}$

Differences between the two study groups with regard to patient characteristics and treatment outcome were assessed with the Fisher exact test and the Maentel-Haensel test. A $\mathrm{p}<0.05$ was considered to be statistically significant.

\section{Results}

Quadruple therapy was given to 82 consecutive $H$ pylori positive patients, who were referred to our centre for diagnostic upper gastrointestinal tract endoscopy. Table 1 gives the demographic and clinical characteristics of the patients. The prevalence of peptic ulcer disease was similar in the two groups. All patients completed the treatment course and underwent control endoscopy to assess cure of $H$ pylori infection. No serious side effects were reported.

Susceptibility testing showed 43 met-S $H$ pylori (group I) and 39 met-R H pylori (group II). All isolated $H$ pylori strains were susceptible to tetracycline.

Cure of $H$ pylori infection could be achieved in $42 / 43$ patients (98\%) in group I and $32 / 39$ patients $(82 \%)$ in group II $(\mathrm{p}=0.02)$. The $H$ pylori eradication efficacy of quadruple therapy is significantly impaired in the met- $R$ group (table 1).

At follow up endoscopy six weeks after the end of the eradication therapy, peptic ulcers were healed in all but one case. The patient with persisting active duodenal ulcer stopped acid suppressive therapy after the end of the eradication treatment. The cultures of the biopsy specimens were negative for $H$ pylori infection, but histological examination of the antrum biopsy samples showed a low number of $H$ pylori. Pretreatment culture showed met-S $H$ pylori. The patient received $\mathrm{H}_{2}$ histamine 
receptor antagonist, and a second control endoscopy 12 weeks after the end of the eradication therapy showed complete ulcer healing and absence of $H$ pylori on both culture and histopathological assessments.

\section{Discussion}

This study compares the efficacy of quadruple therapy in patients infected with either met-R or met-S H pylori in an area with a high prevalence of met-R $H$ pylori. Met-R $H$ pylori were found in $48 \%$ of the patients. Cure of infection was obtained in $98 \%$ of patients infected with met-S $H$ pylori, but in only $82 \%$ of patients infected with met-R strains. This means a significantly impaired eradication efficacy of quadruple therapy in cases of metronidazole resistance.

Resistance to metronidazole, the mainstay of many eradication regimens, is well documented. A multicentre European study on the prevalence of metronidazole resistance in vitro showed that overall $27.5 \%(7-49 \%)$ of the strains tested were resistant. ${ }^{10}$ Amalgamating the results of studies using metronidazolecontaining bismuth triple therapy showed a reduction in efficacy from $92 \%$ (mean) in patients infected with met-S $H$ pylori to $44 \%$ (mean) in patients infected with met-R $H$ pylori. ${ }^{7}$ In the Amsterdam area a similar fall in efficacy was observed from 90 to $38 \%$ using bismuth triple therapy in patients infected with met-S and met-R $H$ pylori respectively. ${ }^{6}$ However, for an unselected population with a background metronidazole resistance of approximately $30 \%$ this would result in a decrease in efficacy of $17-18 \%$ of the eradication regimen for the whole population.

Hosking et al were the first to attempt $H$ pylori eradication with quadruple regimens, ${ }^{9}$ but unfortunately pretreatment metronidazole susceptibility testing was not performed. This study was conducted in an area with assumed high prevalence of resistance to metronidazole, although exact data on metronidazole resistance were missing. The quadruple regimen was effective in $90 \%$, the first indication that this regimen also might be effective in patients harbouring met-R $H$ pylori.

The present data provide further evidence that addition of an acid suppressive agent such as a PPI to the bismuth triple regimen may improve the usually impaired efficacy of this therapy in patients infected with met-R $H$ pylori, as eradication rates of $82 \%$ could be obtained. However, the efficacy of the quadruple regimen is also significantly impaired in cases of metronidazole resistance. De Boer et $a l^{8}$ reported cure in $66 \%$ of patients infected with met-R $H$ pylori; however, these data were derived from a small number of patients (two out of three), from an area with a low prevalence of metronidazole resistance. These early results were obtained in a population of patients with peptic ulcer disease. In such patients compliance is assumed to be excellent because of their more severe dyspeptic complaints as compared with functional dyspeptic patients. ${ }^{13}$ In our study $50 \%$ of the patients had functional dyspepsia, but compliance was still excellent, and patients for whom therapy failed had either functional dyspepsia $(n=5)$ or peptic ulcer disease $(n=3)$.

Recent data from Borody et $a l^{14}$ suggested that met-R $H$ pylori can be eradicated after quadruple therapy with either PPI or $\mathrm{H}_{2}$ histamine receptor antagonist. The study lost some of its power, because 87 of the patients included $(26 \%)$ were lost to follow up endoscopy. Around $23 \%$ of the patients harboured met-R $H$ pylori. Of all the patients who completed follow up, quadruple therapy failed in only three, of whom two were infected with met-R $H$ pylori.

Also Seppala et $a l^{15}$ reported a considerable success rate for a two week quadruple regimen, after failure of bismuth triple therapy. Therapy was successful in $86 \%$ of 49 patients, all harbouring met-R $H$ pylori. As a comparison with the treatment of met-S $H$ pylori was not performed, it remains speculative whether metronidazole resistance impaired efficacy in this study. However, compared with the mean eradication rate of $96 \%{ }^{5}$ for quadruple therapy when given as the initial therapy, the effectiveness is about $10 \%$ lower when used for retreatment.

The exact mechanism by which acid suppression enhances the efficacy of triple therapy in patients infected with met-R $H$ pylori is not known. Although Borody et $a l^{14}$ found better results using PPI than $\mathrm{H}_{2}$ histamine receptor antagonist, it is not known whether this is due to more profound acid suppression or intrinsic PPI related antimicrobial properties. If addition of PPIs does not influence the action of metronidazole in met-R $H$ pylori, regimens containing bismuth, tetracycline, and omeprazole without metronidazole should be as efficacious as the quadruple regimen. In one retreatment study, however, the efficacy of this $\mathrm{PPI} / \mathrm{bismuth} /$ tetracycline regimen was $50-75 \%$ depending on the dose of omeprazole used. ${ }^{16}$ This would suggest a PPI modulated impact of metronidazole on antimicrobial killing in vivo despite resistance to this antimicrobial agent in vitro.

Theoretically, PPI may influence the metabolism of $H$ pylori by the interacting with sulphydryl groups, which are part of many enzyme systems. ${ }^{17}$ Enzymes such as superoxide dismutase and catalase that mediate detoxification of free hydroxyl radicals may be inhibited directly by omeprazole, thereby leading to increased concentrations of these compounds in the presence of metronidazole. ${ }^{18-20}$

From our study with well documented pretreatment susceptibility testing, it appears that eradication efficacy of quadruple therapy is impaired in patients infected with met- $\mathrm{R} H$ pylori. In principle, metronidazole-containing therapy should be avoided in cases of infection with met-R $H$ pylori. If other eradication regimens fail, the combination of PPI, bismuth triple or quadruple therapy without metronidazole remains one of the possible therapeutic modalities for cure of $H$ pylor $i$ infection in areas known for high prevalence of metronidazole resistance. 
1 Van der Hulst RWM, van der Ende A, Dekker FW, et al. Effect of Helicobacter pylori eradication on gastritis in relation to $c a g A$ : a prospective one year follow up study. Gastroenterology 1997;113:25-30.

2 Marshall BJ. Helicobacter pylori. Am $\mathcal{f}$ Gastroenterol 1994;89(suppl):116-28.

3 Van der Hulst RWM, Tytgat GNJ. Helicobacter pylori and peptic ulcer disease. Scand F Gastroenterol 1996;31(supp 220): 10-18.

$4 \mathrm{NIH}$ Consensus Conference. Helicobacter pylori in peptic ulcer disease. ҒAMA 1994;272:65-9.

5 Van der Hulst RWM, Keller JJ, Rauws EAJ, Tytgat GNJ. Treatment of Helicobacter pylori infection: review of the world literature. Helicobacter 1996;1:6-19.

6 Noach LA, Langenberg WL, Bertola MA, et al. Impact of metronidazole resistance on the eradication of Helicobacter pylori. Scand f Infect Dis 1994;26:321-7.

7 Penston JG. Review article: Helicobacter pylori eradication understandable caution but no excuse for inertia. Aliment Pharmacol Ther 1994;8:369-89.

8 de Boer WA, Driessen WMM, Jansz AR, Tytgat GNJ. Effect of acid suppression on efficacy of treatment for Helicoof acid suppression on efficacy of treatment for

bacter pylori infection. Lancet 1995;345:817-20.
9 Hosking SW, Ling TKW, Yung MY, et al. Randomised controlled trial of short term treatment to eradicate Helicobacter pylori in patients with duodenal ulcer. $B M \mathcal{F}$ 1992;305:502-4.

10 European study group on antibiotic susceptibility of Helicobacter pylori: results of a multicentre European survey in 1991 of metronidazole resistance in Helicobacter pylori. Eur 7 Clin Microbiol Infect Dis 1992;11:777-81.

11 Weel JFL, van der Hulst RWM, Gerrits Y, et al. Heterogeneity in susceptibility to metronidazole among Helicobacter pylori isolates from patients with gastritis or peptic ulcer disease. F Clin Microbiol 1996;34:2158-62.

12 Machin D, Campbell MJ. Statistical tables for the design of clinical trials. Oxford: Blackwell Scientific Publications.

13 Graham D, Lew GM, Malaty HM, et al. Factors influencing the eradication of Helicobacter pylori with triple therapy. Gastroenterology 1992;102:493-6.

14 Borody TJ, Andrews P, Fracchia G, et al. Omeprazole enhances efficacy of triple therapy in eradicating Helicobacter pylori. Gut 1995;37:477-81.

15 Seppala K, Sipponen P, Nuutinen $\mathrm{H}$, et al. Intent to treat metronidazole resistant $\mathrm{H}$. pylori infection to $100 \%$ of cure. Therapy with quadruple therapy and its modification. Gastroenterology 1995;108:A216.

16 Carrick J, Lian JX, Daskalopoulos G. Effectiveness of two alternative second line therapies for $\mathrm{H}$. pylori eradication following failure of standard triple therapy [abstract]. $A m \mathcal{F}$ Gastroenterol 1994;89:1366.

17 Wallmark B, Brandstrom A, Larsson $\mathrm{H}$. Evidence for acid-induced transformation of omeprazole into an active inhibitor of $\mathrm{H}^{+}+\mathrm{K}^{+}$-ATP-ase within the parietal cell. Biochim Biophys Acta 1984;778:549-58.

18 Smith MA, Edwards DI. Redox potential and oxygen concentration as factors in susceptibility of Helicobacter pylori to nitrohetrocyclic drugs. F Antimicrob Chemother pylori to nitroh

19 Edwards DI. Nitroimidazole drug action and resistance mechanisms. I. Mechanisms of action. F Antimicrob Chemother 1993;31:9-20.

20 Cederbrant G, Kahlmeter G, L Jungh. Porposed mechanisms for metronidazole resistance in Helicobacter pylori. $\mathcal{F}$ Antimicrob Chemother 1992;29:115-20. 